\title{
Pediatric Crohn's disease with severe morbidity manifested by gastric outlet obstruction: two cases report and review of the literature
}

\author{
Eun Sil Kim, Ji Hyung Park, Yon Ho Choe, Mi Jin Kim \\ Department of Pediatrics, Samsung Medical Center, Sungkyunkwan University School of Medicine, Seoul, Korea
}

Crohn's disease (CD) presenting as gastric outlet obstruction is rare but serious clinical presentation of CD causing severe morbidity. However, there have been few case reports concerning this disorder in East Asian children and adolescents. The current case report describes 2 pediatric patients with $\mathrm{CD}$ who had had gastric outlet obstruction as an initial symptom of CD. Two pediatric patients developed postprandial vomiting, bloating, and unintentional weight loss. The upper endoscopy result indicated that there was pyloric obstruction with mucosal edema, inflammation and ulcers. The serologic test and colonoscopy results suggested CD. These patients were treated with infliximab, and endoscopic balloon dilation without surgery and showed remarkable improvement in obstructing symptoms with maintaining clinical and biochemical remission. This case report elucidates the benefits of early intervention using infliximab and endoscopic balloon dilation to improve gastric outlet obstruction and achieve baseline recovery in patients with upper gastrointestinal B2 phenotype of CD. (Intest Res 2021;19:472-477)

Key Words: Gastric outlet obstruction; Crohn disease; Pediatric

\section{INTRODUCTION}

Crohn's disease (CD), which is a type of inflammatory bowel disease, is a multifactorial idiopathic chronic disease of the gastrointestinal tract. ${ }^{1}$ Approximately $25 \%$ of patients with CD have been diagnosed during childhood and adolescence, and the incidence in pediatric population has increased significantly over the past decades. ${ }^{2}$ In particular, in children with $\mathrm{CD}$, patients had a negative effect on nutrition, growth, adolescent expression, and overall quality of life compared to adult patients. $^{3}$

The large GETAID cohort identified young age, upper gastrointestinal (UGI) and rectal involvement (but not colonic or

Received June 19, 2020. Revised July 31, 2020. Accepted August 7, 2020 Correspondence to Mi Jin Kim, Department of Pediatrics, Samsung Medical Center, Sungkyunkwan University School of Medicine, 81 Irwon-ro, Gangnam-gu, Seoul 06351, Korea. Tel: +82-2-3410-0951, Fax: +82-2-34100043,E-mail:mijin1217.kim@samsung.com ileal), and stricturing or penetrating disease (B2 or B3 phenotype) are bad prognostic factors over 15 years of $\mathrm{CD}^{4}$ Therefore, in pediatric CD patients, it is important to clarify disease phenotype and stratified the risk level and prognostic factors. According to risk stratification of GETAID cohort, pediatric CD presenting as gastric outlet obstruction is important clinical presentation of $\mathrm{CD}$ causing morbidity despite of its rarity.

Most patients with gastroduodenal CD are asymptomatic, but the most common symptom is epigastric pain, which is often alleviated by food and proton pump inhibitors. About $30 \%$ of symptomatic patients with gastroduodenal CD undergo surgery mainly due to gastroduodenal obstruction. Endoscopic intervention, such as endoscopic balloon dilation (EBD), can be performed in selected patients. ${ }^{5}$

Here, we report 2 cases of pediatric CD patient who present gastroduodenal obstructing symptom as an initial symptom of $\mathrm{CD}$ and treated with medication and EBD without surgery. This case report may contribute towards elucidating the bene- 
fits of early intervention using infliximab and EBD to improve gastric outlet obstruction symptoms and achieve baseline recovery in CD patients suffering from gastric outlet obstruction. Moreover, we aimed to undertake a narrative review of the available literature.

\section{CASE REPORT}

This study was approved by the Institutional Review Board of Samsung Medical Center (IRB No. 2020-01-033) and was conducted in accordance with the Declaration of Helsinki. The patient's parents provided written informed consent for the publication.

\section{Case 1}

A 15-year-old male patient was referred to Samsung Medical Center with a 9-month history of nausea, postprandial vomiting, bloating, and unintentional weight loss of about $15 \mathrm{~kg}$ (he lost weight from $53 \mathrm{~kg}$ to $38 \mathrm{~kg}$, about $30 \%$ weight loss from healthy state). He exhibited mild pallor of both conjunctiva and mild joint pain on both ankles during walking around. The rest of the physical examination results were normal.

Nine months prior to referral, esophagogastroduodenoscopy (EGD) demonstrated severe edema and ulcerations on pylorus, suspecting of peptic ulcer disease. Upon admission, the laboratory studies revealed anemia (hemoglobin $10.7 \mathrm{~g} / \mathrm{dL}$ with a mean corpuscular volume $88.6 \mathrm{fL}$, hematocrit 32.5\%) but, albumin and inflammatory markers (erythrocyte sedimentation rate and C-reactive protein) were within the normal limits. The serologic test for autoantibodies revealed the presence of anti-Saccharomyces cerevisiae antibodies was positive, and the anti-neutrophil cytoplasmic antibodies was negative. Stool occult blood was positive and stool calprotectin was over $1,000.0 \mu \mathrm{g} / \mathrm{g}$.

EGD showed reflux esophagitis (Los Angeles classification grade B), multiple esophageal ulcers, edematous mucosa in stomach. A significant narrowing of the pylorus was apparent and distended stomach was filled with food retention due to gastric outlet obstruction (Fig. 1A and B). There were multiple erosions in terminal ileum, swelling of ileocecal valve, but the rest of colon was normal (Fig. 2). Biopsies from EGD revealed focally enhanced gastritis (Fig. 3A) and terminal ileal and colonic biopsies revealed signs of chronic inflammation including villous blunting, crypt distortion, and increased lymphoplasmacytic infiltration (Fig. 3B). Plain X-ray and magnetic resonance enterography showed markedly distended stomach because of pyloric obstruction (Fig. 4).

Based on the laboratory, endoscopic and radiologic tests, he was diagnosed with $\mathrm{CD}$. The patient with $\mathrm{CD}$-related gastric outlet obstruction, was initially treated with methylpredniso-
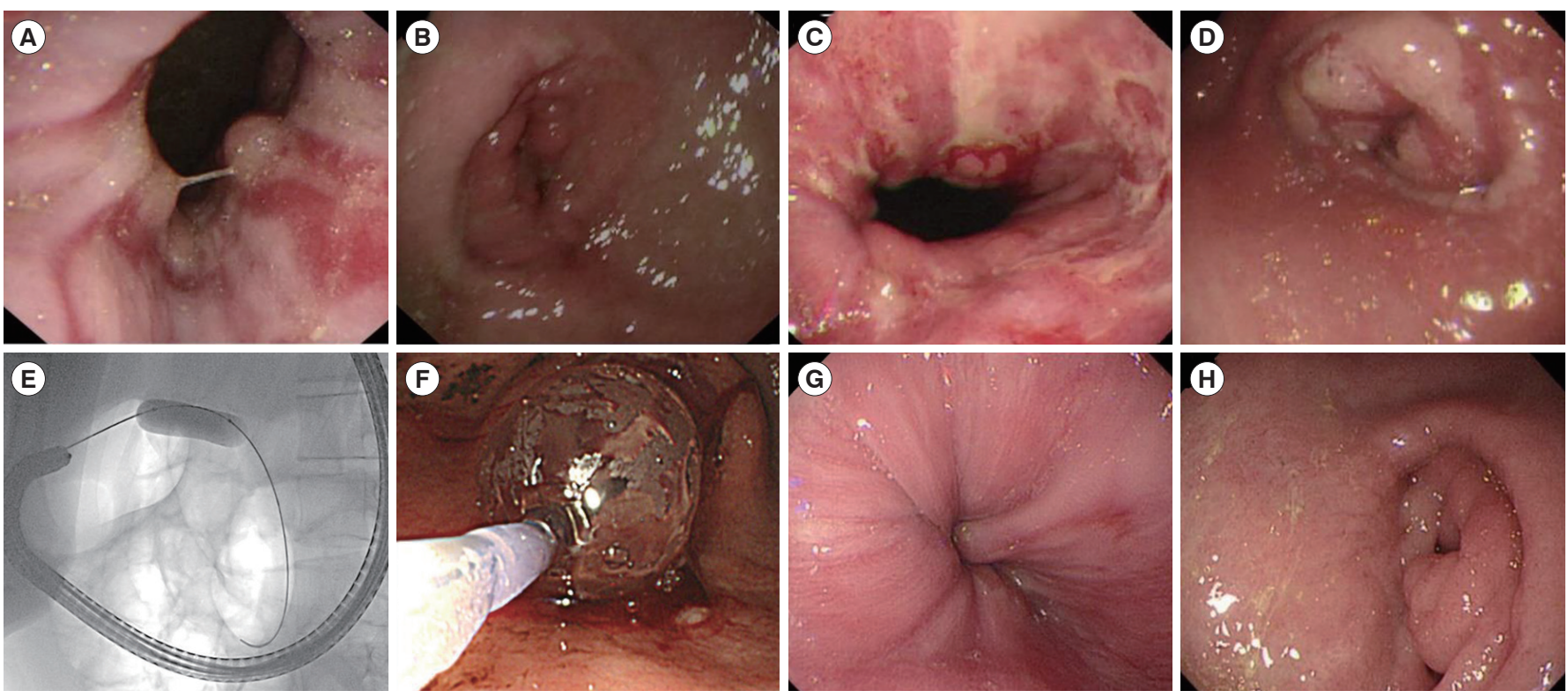

Fig. 1. Esophagogastroduodenoscopy at diagnosis (case 1). $(A, B)$ Upper endoscopic findings at referral. Reflux esophagitis at low esophagus, multiple esophageal ulcers, and significant narrowing of pylorus. (C, D) Follow-up upper endoscopic findings after 11 weeks. Apparent pyloric obstruction and multiple ulcerations on antrum. (E, F) Fluoroscopically guided balloon dilation. $(G, H)$ Follow-up upper endoscopic findings after 20 weeks. Remarkable improvement of reflux esophagitis and pyloric obstruction. 
lone $20 \mathrm{mg}$ twice a day ( $1 \mathrm{mg} / \mathrm{kg} /$ day) for a week and then slowly tapered off for a month. He was prescribed azathioprine $50 \mathrm{mg}$ once a day and mesalazine $1-0.5 \mathrm{~g}$. Simultaneously, he started exclusive enteral nutrition for treatment of $\mathrm{CD}$ and prevention of aggravation of obstructing symptom.
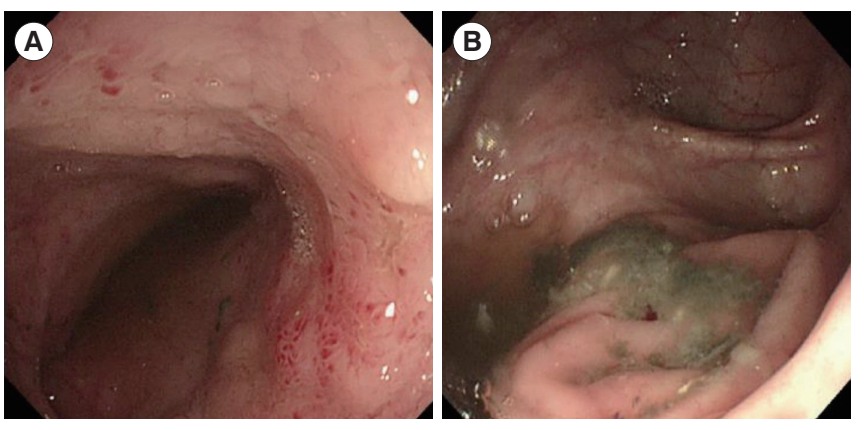

Fig. 2. Colonoscopy at referral (case 1). (A) Multiple erosions in terminal ileum. (B) Mild swelling in ileocecal valve.
Six weeks after diagnosis, there was a partial stricture confirmed on EGD with ongoing symptoms of gastric outlet obstruction prompting commencement of infliximab $(5 \mathrm{mg} / \mathrm{kg})$. Despite of medical treatment, he revisited emergency room for aggravation of postprandial vomiting and epigastric pain at 11 weeks after diagnosis. EGD revealed that gastric outlet obstruction was severe as the scope was not able to be intubated to duodenum (Fig. 1C and D). With fluoroscopic guidance, multifunctional coil catheter was passed guide wire to the distal part of the stricture and then coil catheter was removed. A pyloric dilator with a balloon size of $15 \mathrm{~mm}$ was passed over the guide wire and employed for 1 minute followed pyloric dilator with $18 \mathrm{~mm}$-sized-balloon for 1 minute (Fig. $1 \mathrm{E}$ and F). Ten months after EBD, the patient maintained clinical and biochemical remission on partial enteral nutrition and infliximab therapy, with no demonstrable recurrence of pyloric stenosis (Fig. $1 \mathrm{G}$ and H).
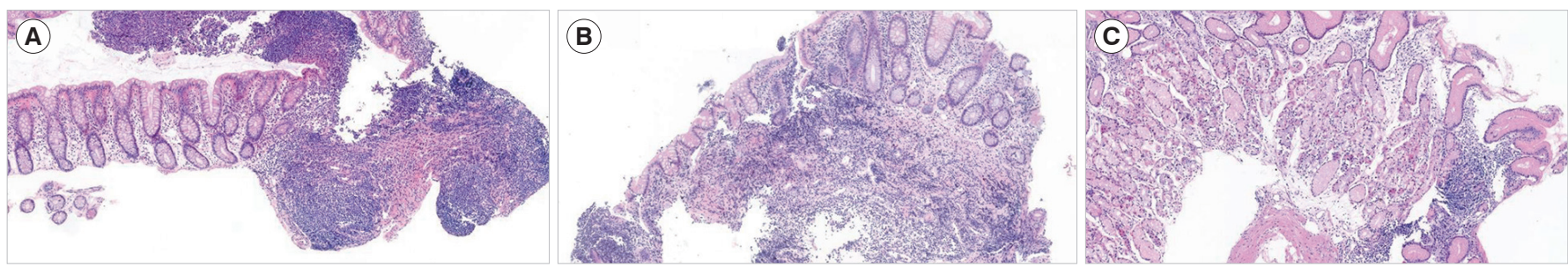

Fig. 3. Histopathologic findings of cases. (A) Case 1: gastric biopsy specimens showing small clusters of lymphocytes in the lamina propria, also called as focally enhanced gastritis $\left(H \& E_{1} \times 4\right)$. (B) Case 1: terminal ileal biopsy specimens showing villous blunting, crypt distortion and increased lymphoplasmacytic infiltration $\left(H \& E_{1} \times 4\right)$. (C) Case 2: colonic biopsy specimens showing a shallow ulcerative lesion and increased lymphoplasmacytic infiltration in submucosal layer and lamina propria of mucosa $\left(H \& E_{1} \times 4\right)$.
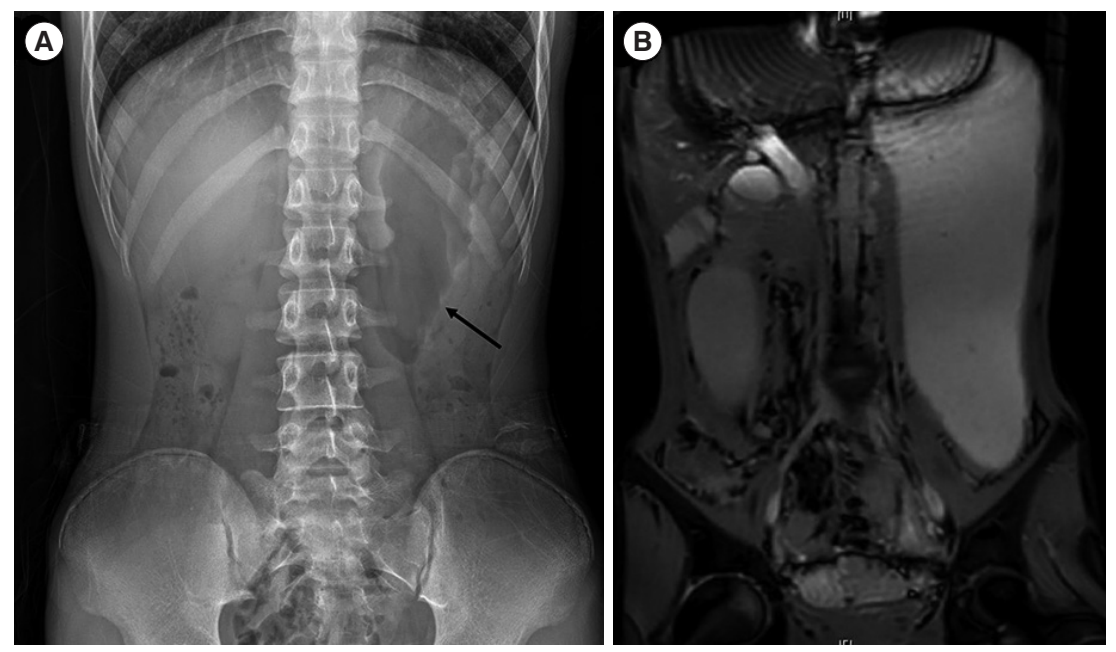

Fig. 4. Abdomen imaging at referral (case 1). (A) Plain X-ray revealed severe distended stomach (arrow) due to obstruction. (B) Magnetic resonance enterography revealed markedly distended stomach with gastric outlet obstruction, and limited evaluation of small bowel loops because of poor passage of contrast and collapse of small bowel. 


\section{Case 2}

A 14-year-old male patient presented with abdominal pain, watery diarrhea, anorexia, and history of postprandial vomiting for 2 months. Four months ago, he was admitted to other hospital where he received EGD and was diagnosed with peptic ulcer disease. At that time, he treated with antacids for pyloric ulcer and balloon dilation for pyloric stenosis. Four months later, he complained postprandial vomiting, fever, unintentional weight loss about $14 \mathrm{~kg}$ (he lost weight from $59 \mathrm{~kg}$ to $45 \mathrm{~kg}$, about $24 \%$ weight loss from healthy state) again, and he referred to Samsung Medical Center for evaluation and management.

The laboratory studies revealed elevated inflammatory markers, erythrocyte sedimentation rate and C-reactive protein were $36 \mathrm{~mm} / \mathrm{hr}$ and $18.56 \mathrm{mg} / \mathrm{L}$, respectively. EGD demonstrated edematous mucosa in stomach, multiple pyloric ulcers, and deformity with a significant narrowing of the pylorus (Fig. 5A). There were also several multiple aphthous ulcers in terminal ileum, and cecum to sigmoid colon (Fig. 5B). Histopathologic examination of gastric and duodenal specimens demonstrated chronic active gastritis with inflamed granulation tissue and colonic specimens revealed ulcerative lesions and lymphoplasmacytic infiltration in submucosal layer and lamina propria (Fig. 3C). Under diagnosis of $\mathrm{CD}$, he was treated with azathioprine $50 \mathrm{mg}$ once a day and mesalazine $1 \mathrm{~g}$ twice a day simultaneously with exclusive enteral nutrition for treatment CD.

After 1-year, persistent gastric outlet obstruction was confirmed via EGD with ongoing symptoms of epigastric pain and vomiting prompting EBD and commencement of infliximab. He remained clinical and biochemical remission for a further 5 years, with no demonstrable recurrence of pyloric stenosis.

\section{DISCUSSION}

According to recently published literatures, pediatric CD involving UGI tract has become increasingly recognized by the introduction of routine upper endoscopy and biopsies for all children and adolescent being evaluated for $\mathrm{CD}^{6}{ }^{6}$ Studies in pediatric patients who had simultaneous EGD at the time of diagnosis revealed that the prevalence of gastroduodenal involvement of $\mathrm{CD}$ is up to $36 \%-53 \% .^{7.8}$ According to this result, the surveillance of UGI involvement is conducted as a significant factor in diagnosis, risk and prognostic evaluation of CD. Patient with UGI manifestations have a higher risk of more aggressive stricturing and more penetrating form of the disease, meaning more recurrences, frequent hospitalization, and more surgical intervention. ${ }^{9}$

The gold standard of diagnosis of UGI-CD is endoscopy with biopsy, which is ideal for surveying the mucosa and is most likely to identify early gastric and/or duodenal involvement. Endoscopic findings of UGI-CD include patchy erythema, mucosal friability, thickened folds, ulcerations, and in severe cases strictures, luminal narrowing, fistula of UGI tract. UGI-CD is relatively rare disease, thus other possible etiologies of gastric outlet obstruction such as peptic ulcer disease, Menetrier disease, collagen vascular disease, various infections like Helicobacter pylori, cytomegalovirus and Mycobacterium tuberculosis, nonsteroidal anti-inflammatory drug use, malignant and infiltrative disease should be ruled out through imaging or upper endoscopy. ${ }^{10}$

One-third of patients with isolated UGI-CD do not have small or large intestine disease at the time of diagnosis of CD but develop distal disease over time. Also, only $4 \%$ to $11 \%$ of patients with UGI-CD have granulomas in biopsies, making
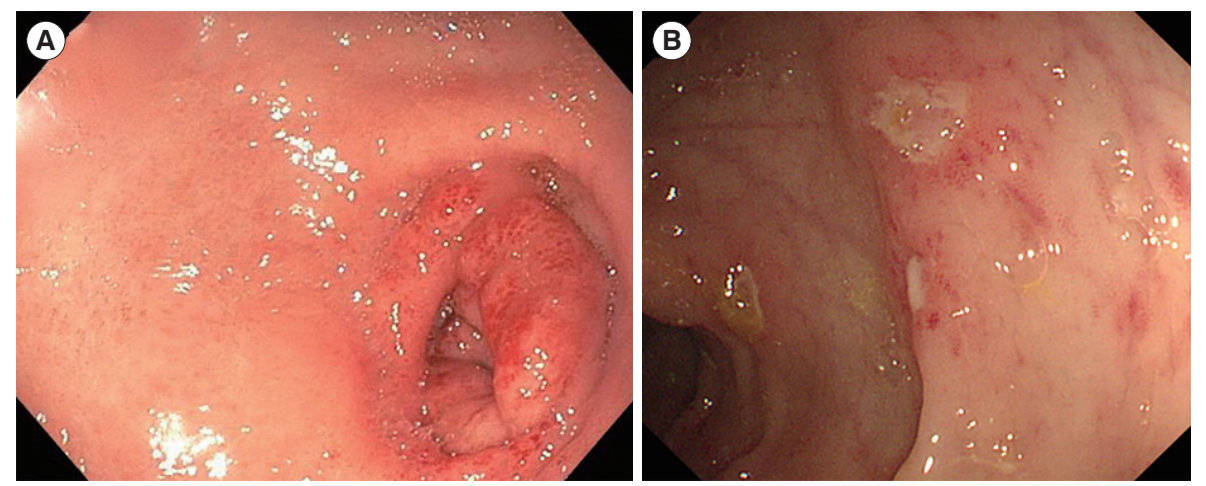

Fig. 5. Esophagogastroduodenoscopy and colonoscopy at referral (case 2). (A) Upper endoscopic findings at referral. Edematous mucosa, multiple pyloric ulcers, and significant narrowing of pylorus. (B) Several multiple aphthous ulcers in terminal ileum, and cecum to sigmoid colon. 
the diagnosis of atypical isolated gastric CD more challenging. ${ }^{5}$ Although the patient in case 1 had symptoms related with isolated UGI-CD, the colonoscopy findings revealed that there were also involvements of small and large intestine as well as gastric outlet obstruction on EGD. According to $\mathrm{Nu}-$ gent and Roy, ${ }^{11} 92 \%$ of patients diagnosed with UGI-CD had developed distal CD within a follow-up of 11.7 years. Therefore, our patient has high potency of progress to distal CD.

An extensive English literature search revealed that only 1 previously reported pediatric CD patient with initial presentation of gastric outlet obstruction. ${ }^{12}$ Here, we add 2 cases of CD patients who were mistaken for peptic ulcer disease and had gastroduodenal obstructing symptoms as an initial presentation of CD treated with medication and EBD without surgery.

In UGI-CD patients, the obstruction is important prognostic factor determining operative intervention and related high failure rates of medical management. ${ }^{13,14}$ Also, because of frequently coexisting with ileocolic CD and underlying tissue inflammation and fibrosis, there is scare data on specific management of gastric outlet obstructing $\mathrm{CD}$ which is challenging to physicians. Management of gastric outlet obstructing CD can be divided into medical and surgical treatment. Even though there are debates regarding to long-term efficacy of EBD, medical treatment such as proton pump inhibitors for symptomatic relief, steroids to target any inflammatory component, infliximab to reduce inflammation-mediated edema, and endoscopic intervention such as EBD can be considered to treat gastroduodenal strictures in CD patients before surgery. ${ }^{15}$

Systemic steroids are one of the most important treatment options. One case report demonstrated the use of intralesional steroids ${ }^{16}$ and the other reported the use of inhaled steroids in the treatment of isolated gastric $\mathrm{CD} .{ }^{17}$ Azathioprine, methotrexate and 6-mercaptopurine have been described to maintain remission and should be instituted early in the disease course. ${ }^{15}$ The efficacy of infliximab (anti-tumor necrosis factor) administration for UGI-CD was reported to improve disease activity scores and symptoms, however, the effect of antitumor necrosis factor in gastric outlet obstructing $\mathrm{CD}$ remains to be demonstrated. There is only 1 case report described a remarkable response to infliximab, ${ }^{18}$ and another case report described successful treatment of pyloric obstructing CD by subcutaneous injection of adalimumab. ${ }^{19}$ Endoscopic interventions for gastric outlet obstruction include intralesional steroid injection, EBD, and stent insertion. There have been no controlled studies about effectiveness of these interventions in pyloric stenosis of CD. Recently, a few literatures revealed that EBD seems to be a useful tool for management of pyloric stricture in $\mathrm{CD}$ and is a viable alternative to open surgery. ${ }^{20} \mathrm{It}$ has been reported that about $30 \%$ in patients with EBD experienced recurrence, but 1-month response could serve as an early predictor of long-term response. ${ }^{20}$ Therefore, surgical treatment in gastric outlet obstructing CD could be reserved for harsh conditions such as failure to balloon dilatation. These case reports may contribute towards elucidating the benefits of early intervention using steroid, infliximab and endoscopic intervention to improve gastric outlet obstruction symptoms, and achieve baseline recovery in patients with CD. In addition, among patients with gastric outlet obstruction who have severe inflammation in pylorus, if EBD is done immediately, it is highly likely that obstruction will recur again due to inflammation.

Here, we report 2 cases of gastric outlet obstruction which type is proven to be bad prognosis of pediatric CD. This case report may contribute towards elucidating the benefits of early intervention using steroid, infliximab and endoscopic intervention to improve gastric outlet obstruction symptom, and achieve baseline recovery in patients with pediatric CD patients, suffering from gastric outlet obstruction. Therefore, surgical therapy in gastric outlet obstructing CD should be reserved for severe conditions such as unsuccessful balloon dilatation.

In conclusion, although there is the serious morbidity, the management of gastric outlet obstruction in pediatric UGI-CD patients is overlooked in consensus guidelines. The appropriate medical and surgical management require further research.

\section{ADDITIONAL INFORMATION}

\section{Funding Source}

The authors received no financial support for the research, authorship, and/or publication of this article.

\section{Conflict of Interest}

No potential conflict of interest relevant to this article was reported.

\section{Author Contribution}

Conceptualization: Kim ES, Choe YH, Kim MJ. Methodology: Kim ES, Choe YH, Kim MJ. Formal analysis: Kim ES, Park JH, Choe YH, Kim MJ. Writing-original draft: Kim ES. Writing-re- 
view and editing: Kim ES, Choe YH, Kim MJ. Approval of final manuscript: all authors.

\section{Others}

We thank the parents of the patient for permitting us to use the patients' data.

\section{ORCID}

Kim ES

Park JH

Choe $\mathrm{YH}$

Kim MJ

\section{REFERENCES}

1. Hyams JS. Inflammatory bowel disease. Pediatr Rev 2005;26: 314-320.

2. Benchimol EI, Fortinsky KJ, Gozdyra P, Van den Heuvel M, Van Limbergen J, Griffiths AM. Epidemiology of pediatric inflammatory bowel disease: a systematic review of international trends. Inflamm Bowel Dis 2011;17:423-439.

3. Chouliaras G, Margoni D, Dimakou K, Fessatou S, Panayiotou I, Roma-Giannikou E. Disease impact on the quality of life of children with inflammatory bowel disease. World J Gastroenterol 2017;23:1067-1075.

4. Cosnes J, Bourrier A, Nion-Larmurier I, Sokol H, Beaugerie L, Seksik P. Factors affecting outcomes in Crohn's disease over 15 years. Gut 2012;61:1140-1145.

5. Horjus Talabur Horje CS, Meijer J, Rovers L, van Lochem EG, Groenen MJ, Wahab PJ. Prevalence of upper gastrointestinal lesions at primary diagnosis in adults with inflammatory bowel disease. Inflamm Bowel Dis 2016;22:1896-1901.

6. Levine A, Koletzko S, Turner D, et al. ESPGHAN revised porto criteria for the diagnosis of inflammatory bowel disease in children and adolescents. J Pediatr Gastroenterol Nutr 2014; 58:795-806.

7. Abuquteish D, Putra J. Upper gastrointestinal tract involvement of pediatric inflammatory bowel disease: a pathological review. World J Gastroenterol 2019;25:1928-1935.

8. Tobin JM, Sinha B, Ramani P, Saleh AR, Murphy MS. Upper gastrointestinal mucosal disease in pediatric Crohn disease and ulcerative colitis: a blinded, controlled study. J Pediatr Gastroenterol Nutr 2001;32:443-448.

9. Song XM, Gao X, Li MZ, et al. Clinical features and risk factors for primary surgery in 205 patients with Crohn's disease: analysis of a South China cohort. Dis Colon Rectum 2011;54: 1147-1154.

10. Inayat F, Ullah W, Hussain Q, Shafique K. Crohn's disease presenting as gastric outlet obstruction: a therapeutic challenge? BMJ Case Rep 2017;2017:bcr2016218181.

11. Nugent FW, Roy MA. Duodenal Crohn's disease: an analysis of 89 cases. Am J Gastroenterol 1989;84:249-254.

12. Ho CH, Sinatra FR, Pietzak MM. A 9-year-old girl with poor weight gain and postprandial vomiting. Gastroenterology 2015;148:904-905.

13. Murray JJ, Schoetz DJ Jr, Nugent FW, Coller JA, Veidenheimer MC. Surgical management of Crohn's disease involving the duodenum. Am J Surg 1984;147:58-65.

14. Yamamoto T, Fazio VW, Tekkis PP. Safety and efficacy of strictureplasty for Crohn's disease: a systematic review and metaanalysis. Dis Colon Rectum 2007;50:1968-1986.

15. Tremaine WJ. Gastroduodenal Crohn's disease: medical management. Inflamm Bowel Dis 2003;9:127-128.

16. Balendran K, Udumalagala S, Nawaraththne NMM. Pyloric stenosis as a manifestation of isolated gastric Crohn's disease responding to intralesional steroid injection and balloon dilation: a case report. J Med Case Rep 2019;13:331.

17. Ibrahim SH, Smyrk TC, Faubion WA. Treatment of isolated gastric Crohn's disease with inhaled corticosteroids. Case Rep Gastroenterol 2008;2:363-368.

18. Odashima M, Otaka M, Jin M, et al. Successful treatment of refractory duodenal Crohn's disease with infliximab. Dig Dis Sci 2007;52:31-32.

19. Gaggar S, Scott J, Thompson N. Pyloric stenosis associated Crohn's disease responding to adalimumab therapy. World J Gastrointest Pharmacol Ther 2012;3:97-99.

20. Matsui T, Hatakeyama S, Ikeda K, Yao T, Takenaka K, Sakurai T. Long-term outcome of endoscopic balloon dilation in obstructive gastroduodenal Crohn's disease. Endoscopy 1997; 29:640-645. 